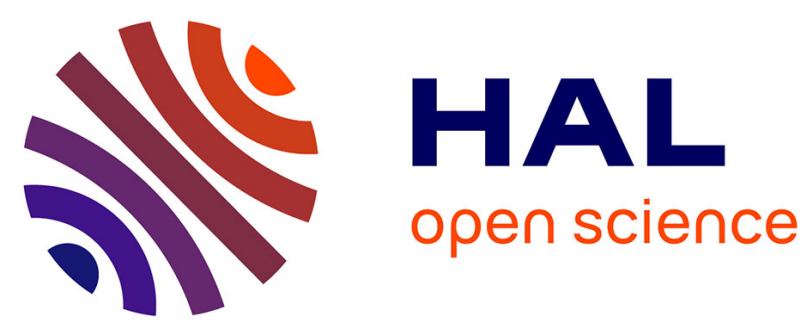

\title{
Pirfenidone photosensitization in patients with idiopathic pulmonary fibrosis a case series
}

\author{
C Droitcourt, Henri Adamski, A Polat, E Polard, M Kerjouan, B Arnouat, M
}

Le Garrec, E Oger, A Dupuy, S Jouneau

\section{- To cite this version:}

C Droitcourt, Henri Adamski, A Polat, E Polard, M Kerjouan, et al.. Pirfenidone photosensitization in patients with idiopathic pulmonary fibrosis a case series. British Journal of Dermatology, 2018, 178 (3), pp.e222-e223. 10.1111/bjd.16016 . hal-01771415

HAL Id: hal-01771415 https://hal-univ-rennes1.archives-ouvertes.fr/hal-01771415

Submitted on 26 Apr 2018

HAL is a multi-disciplinary open access archive for the deposit and dissemination of scientific research documents, whether they are published or not. The documents may come from teaching and research institutions in France or abroad, or from public or private research centers.
L'archive ouverte pluridisciplinaire HAL, est destinée au dépôt et à la diffusion de documents scientifiques de niveau recherche, publiés ou non, émanant des établissements d'enseignement et de recherche français ou étrangers, des laboratoires publics ou privés. 
DR HENRI ADAMSKI (Orcid ID : 0000-0001-9117-9306)

Article type : Research Letter

Pirfenidone photosensitization in patients with idiopathic pulmonary fibrosis: a case series

Running head: Pirfenidone photosensitization

C. Droitcourt ${ }^{1,2,3^{*},}$ H. Adamski ${ }^{1 *}$, A. Polat ${ }^{1}$, E. Polard ${ }^{4}$, M. Kerjouan ${ }^{5}$, B. Arnouat ${ }^{5}$, M. Le Garrec ${ }^{5}$, E. Oger ${ }^{3,4}$, A. Dupuy ${ }^{1,3}$, S. Jouneau ${ }^{5,6}$

Affiliations:

1 Department of Dermatology, Rennes University Hospital, Rennes, France

2 Inserm CIC 1414, Rennes, France

3 UPRES-EA-7449 REPERES, Rennes, France

4 Centre of Pharmacovigilance, Rennes University Hospital, Rennes, France

5 Department of Respiratory Diseases, Rennes University Hospital, Rennes, France

6 IRSET UMR1085, Rennes 1 University, Rennes, France

*Equal contribution

Correspondence to: Dr Henri Adamski, Department of Dermatology, Pontchaillou Hospital, 2 rue Henri le Guilloux 35000 Rennes, France, Tel: 003329928 43 49, Fax: 003329928 41 00, E-mail : henri.adamski@chu-rennes.fr

Funding sources: None

Conflicts of interest: Prof S. Jouneau has acted as Investigator, Consultant or in another capacity from Laboratoire Roche. 
The oral antifibrotic agent, pirfenidone (PFD), 5-methyl-1-phenyl-[1H]-pyridine, is used to treat idiopathic pulmonary fibrosis (IPF), a chronic and fatal lung disease. In trials, PFD reduces disease progression and decreases mortality. The most common side events of PFD are skin manifestations $(25 \%)$, described as a photosensitivity or rash, but they are not well characterised ${ }^{1}$. The objective of the present real-life study was to address the question of skin manifestations in patients treated with PFD for IPF.

We performed a single-centre cross-sectional study of 54 patients treated with PFD for IPF (85\% men, median age 74 years, median exposure time 11.9 months), in the Department of Pulmonology (Competence Centre for Rare Lung Diseases), at Rennes University Hospital (CHU), France, between April 2014 and January 2017. The study was approved by the CHU Ethics Committee and all patients signed informed consent in accordance with the principles of the Helsinki Declaration.

Of the 54 patients treated with PFD, 13 (22.2\%) experienced skin manifestations. All were declared to the Rennes Pharmacovigilance. This database showed that 12 patients had photosensitivity and one urticaria. Eight patients, none of whom had a history of photosensitive diseases, were assessed by a dermatologist (Table 1). The mean duration between starting PFD and a skin manifestation was 5.5 months. They developed burning erythema followed by hyperpigmentation which was sharply limited to sun-exposed areas (bald head, face, neck, upper chest and/or dorsa of forearms and hands), where sunscreen has not been applied one day after UV exposure. These findings were consistent with a moderate phototoxic reaction. Skin biopsies performed in cases 1, 3 and 6, showed epidermal spongiosis with a lichenoid reaction and moderate dermal perivascular lymphocytic infiltration. Apoptotic keratinocytes were observed in case 1. All patients were successfully treated with topical corticosteroid within 8 days. Three patients discontinued PFD due to gastrointestinal disorders and fatigue. No patient relapsed. Other long-term medication was continued.

Photobiological explorations were realized on the back of patients with an ultraviolet (UV)A lamp (Waldmann ${ }^{\circledR}$ 182, Reischtett, France) and a solar simulator (Dermolum UM-UW Müller Elektronik ${ }^{\circledR}$, Moosinning, Germany) emitting polychromatic spectrum (95\%UVA/5\%UVB). Polychromatic minimal erythema dose (MED) was evaluated 24 hours after exposure for 5 patients tested in normal values. UVA MED was normal $\left(>20 \mathrm{~J} / \mathrm{cm}^{2}\right)$ at baseline in all of the 3 cases evaluated. After skin reaction, the reactivity threshold was lower in UVA: an erythema appeared for $20 \mathrm{~J} / \mathrm{cm}^{2} 24$ hours after exposure in 6 of 6 patients tested. We examined 5 patients using PFD photopatches (contents of Esbriet ${ }^{\circledR} 267 \mathrm{mg}$ capsule, $30 \%$ petrolatum). The irradiated site of 4 patients was positive one and two days after UVA-irradiation $\left(7 \mathrm{~J} / \mathrm{cm}^{2}\right)$ with 3 having strong crescendo eczematous reaction. The nonirradiated patch showed no reaction. 
Porphyrins in the blood and urine were assayed at PFD introduction and during the skin manifestations in 3 patients: all were normal. The niacin values of 2 of the 3 patients tested were initially low and were not significantly altered after photosensitivity.

To our knowledge, this study represents the largest documented series of PFD photosensitivity because such sporadic case reports have been only published. ${ }^{2-6}$ One fifth of our patients were photosensitive, consistent with data from PFD safety analysis ${ }^{1}$. Our patients seen by a dermatologist had clinical features of phototoxicity. All were treated with maximum dose of PFD. Photobiochemical studies demonstrated the phototoxicity of PFD ${ }^{7}$, confirmed by clinical reported cases. ${ }^{2,3}$ Our results do not indicate that phototoxicity is linked to the metabolism of porphyrins or niacin. In patients with low niacin serum concentration, we did not assess their diets and found no drug-induced niacin deficiency.

Furthermore, three cases of PFD photoallergic reaction were recently published. ${ }^{4,6}$ Photoallergic dermatitis is characterised by eczematous eruption starting in light-exposed areas and later spreading to covered sites. This clinical presentation was not found in our patients, but histology (lichenoid pattern) and photopatch testing (crescendo eczematous reaction) were in accordance with photoallergic features. Therefore, we believe the mechanism underlying the PFD photosensitivity involves a combination of photoallergic and phototoxic effects.

Our photobiological explorations showed that UVA irradiation influenced PFD photosensitivity, as in most drug-induced photosensitization. ${ }^{8}$ Very few cases with PFD phototesting have been reported. ${ }^{3,6}$ In one case, UVA and UVB MEDs were decreased. ${ }^{3}$ Lastly, only one patient had UVA PFD patch and was positive ${ }^{6}$, as in most of our cases tested.

The great photosensitivity of PFD requires optimal management including photoprotection and a close collaboration between dermatologists, pulmonologists and general practitioners.

\section{References}

1. Lancaster L, Albera C, Bradford WZ et al. Safety of pirfenidone in patients with idiopathic pulmonary fibrosis: integrated analysis of cumulative data from 5 clinical trials. BMJ Open Respir Res 2016; 3:e000105.

2. Papakonstantinou E, Prasse A, Schacht V, Kapp A, Raap U. Pirfenidone-induced severe phototoxic reaction in a patient with idiopathic lung fibrosis. J Eur Acad Dermatol Venereol 2016; 30:1354-6. 
3. Tsuruta A, Washio K, Fukunaga A, Nishigori C. Pirfenidone-induced photoleukomelanoderma in a patient with idiopathic pulmonary fibrosis. J Dermatol 2016; 43:207-9.

4. Reinholz M, Eder I, Przybilla B et al. Photoallergic contact dermatitis due to treatment of pulmonary fibrosis with pirfenidone. J Eur Acad Dermatol Venereol 2016; 30:370-1.

5. Caruana DM, Wylie G. Cutaneous reactions to pirfenidone: a new kid on the block. Br J Dermatol 2016; 175:425-6.

6. Park MY, Shim WH, Kim JM, Kim GW, Kim HS, Ko HC, Kim MB, Kim BS. Pirfenidoneinduced photo-allergic reaction in a patient with idiopathic pulmonary fibrosis. Photodermatol Photoimmunol Photomed 2017 Mar 4.

7. Seto $\mathrm{Y}$, Inoue $\mathrm{R}$, Kato $\mathrm{M}$ et al. Photosafety assessments on pirfenidone: photochemical, photobiological, and pharmacokinetic characterization. J Photochem Photobiol 2013; 120:44-51.

8. Khandpur S, Porter RM, Boulton SJ, Anstey A. Drug-induced photosensitivity: new insights into pathomechanisms and clinical variation through basic and applied science. Br J Dermatol 2017; 176: 902-909.

\section{Acknowledgments}

The English text was edited by Dr Owen Parkes

\section{Table}

Table 1. Clinical, biological, and photobiological characteristics of skin manifestations for patients with idiopathic pulmonary fibrosis treated with pirfenidone and seen by a dermatologist. 
Table 1. Clinical, biological and photobiological characteristics of skin manifestations for patients with idiopathic pulmonary fibrosis treated with pirfenidone and seen by a dermatologist.

\begin{tabular}{|c|c|c|c|c|c|c|c|c|c|c|c|c|c|c|}
\hline Case & $\begin{array}{l}\text { Age } \\
\text { (y) }\end{array}$ & Sex & $\begin{array}{l}\text { Phototype } \\
\text { (Fitzpatrick } \\
\text { 's } \\
\text { classificatio } \\
\text { n) }\end{array}$ & Long-term therapy* & $\begin{array}{l}\text { Type of skin side } \\
\text { event and } \\
\text { dermatological } \\
\text { examination }\end{array}$ & $\begin{array}{l}\text { Period of use } \\
\text { PFD before } \\
\text { skin event, in } \\
\text { months }\end{array}$ & $\begin{array}{l}\text { PFD dose on } \\
\text { onset of } \\
\text { photosensitivity } \\
\text { (mg/day) }\end{array}$ & Treatment & $\begin{array}{l}\text { Polychromatic } \\
\text { MED (Normal > } \\
1 \text { J/cm²) before/ } \\
\text { during PFD } \\
\text { treatment }\end{array}$ & $\begin{array}{l}\text { UVA phototest } \\
\text { before PFD } \\
\text { starting }(20 \\
\text { J/(cm²)** }\end{array}$ & 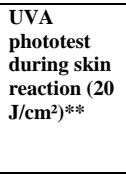 & $\begin{array}{l}\text { PFD } \\
\text { patch } \\
\text { test }\end{array}$ & $\begin{array}{l}\text { Niacin dosage } \\
\text { before/during } \\
\text { PFD treatment } \\
\text { (Normal }>38 \\
\mu \mathrm{mol} / \mathrm{L})\end{array}$ & $\begin{array}{l}\text { Porphyrins } \\
\text { dosage in } \\
\text { blood and } \\
\text { urine } \\
\text { before/during } \\
\text { PFD } \\
\text { treatment } \\
\end{array}$ \\
\hline 1 & 74 & $\mathrm{M}$ & IIII & $\begin{array}{l}\text { Lansoprazole, irbesartan, } \\
\text { rosuvastatin }\end{array}$ & $\begin{array}{l}\text { Phototoxicity } \\
\text { (Grade II) on head } \\
\text { and dorsa of hands }\end{array}$ & 4 & 2403 & $\begin{array}{l}\text { Topical } \\
\text { corticosteroids, } \\
\text { photoprotective } \\
\text { measures, } \\
\text { PFD continued }\end{array}$ & ND & ND & ND & ND & ND & ND \\
\hline 2 & 68 & $\mathrm{M}$ & III & $\begin{array}{l}\text { Lansoprazole, prednisone, } \\
\text { simvastatin, valsartan, } \\
\text { budesonide/formoterol }\end{array}$ & $\begin{array}{l}\text { Phototoxicity } \\
\text { (Grade II) on dorsa of } \\
\text { hands }\end{array}$ & 10 & 2403 & $\begin{array}{l}\text { Photoprotective } \\
\text { measures, } \\
\text { PFD continued }\end{array}$ & Normal: $1.5 / 1.25$ & - & Erythema & $\begin{array}{l}\text { NI :- } \\
\text { UVA: + }\end{array}$ & $40 / 52$ & $\begin{array}{l}\text { Normal// } \\
\text { Normal }\end{array}$ \\
\hline 3 & 77 & F & III & $\begin{array}{l}\text { Amlodipine, salbutamol, } \\
\text { indacatero/glycopyrronium } \\
\text {, alendronic acid, } \\
\text { metoclopramide }\end{array}$ & $\begin{array}{l}\text { Phototoxicity } \\
\text { (Grade II) on } \\
\text { forehead and dorsa of } \\
\text { hands }\end{array}$ & 3 & 2403 & $\begin{array}{l}\text { High potent topical } \\
\text { corticosteroids, } \\
\text { photoprotective } \\
\text { measures. } \\
\text { Decrease PrD dose } \\
\text { (1602mg) due to } \\
\text { digestive disorders }\end{array}$ & $\mathrm{ND}$ & - & $\begin{array}{l}\text { Erythema } \\
\end{array}$ & ND & $22 / 31$ & $\begin{array}{l}\text { Normal/ } \\
\text { Normal }\end{array}$ \\
\hline 4 & 68 & $\mathrm{M}$ & II & $\begin{array}{l}\text { Lercanidipine, olmesartan, } \\
\text { fenofibrate, lansoprazole }\end{array}$ & $\begin{array}{l}\text { Phototoxicity } \\
\text { (Grade II) on head } \\
\text { and neck }\end{array}$ & 11 & 2403 & $\begin{array}{l}\text { Photoprotective } \\
\text { measures } \\
\text { PFD continued }\end{array}$ & ND/Normal: 1.25 & ND & $\begin{array}{l}\text { Erythema } \\
\end{array}$ & $\begin{array}{l}\mathrm{N} \text { :- } \\
\text { UVA :- }\end{array}$ & ND/35 & ND/Normal \\
\hline 5 & 75 & $\mathrm{M}$ & II & $\begin{array}{l}\text { Ramipril/hydrochlorothiazi } \\
\text { de, acetylsalicylate, } \\
\text { bosiprolol, lansoprazole, } \\
\text { rosuvastatin, amlodipine }\end{array}$ & $\begin{array}{l}\text { Phototoxicity } \\
\text { (Grade II) on head, } \\
\text { neck, scalp, ears, and } \\
\text { dorsum of hands }\end{array}$ & 5 & 2403 & $\begin{array}{l}\begin{array}{l}\text { Topical } \\
\text { corticosteroids,PFD } \\
\text { discontinued }\end{array} \\
\end{array}$ & ND/Normal: 1.75 & - & Erythema & $\begin{array}{l}\text { NI: - } \\
\text { UVA: ++ }\end{array}$ & $22 / 51$ & $\begin{array}{l}\text { Normal// } \\
\text { Normal }\end{array}$ \\
\hline 6 & 79 & $\mathrm{M}$ & II & $\begin{array}{l}\text { Acetylsalicylate, } \\
\text { hydrocortisone, } \\
\text { levothyroxine, testostérone, } \\
\text { bisoprolol, atorvastatin, } \\
\text { ramipril, lansoprazole }\end{array}$ & $\begin{array}{l}\text { Phototoxicity } \\
\text { (Grade II) on head, } \\
\text { neck and dorsa of } \\
\text { hands }\end{array}$ & 2 & 2403 & $\begin{array}{l}\text { Topical } \\
\text { corticosteroids, } \\
\text { PFD discontinued }\end{array}$ & ND/Normal: 1.75 & ND & Erythema & $\begin{array}{l}\text { NI: :- } \\
\text { UVA :++ }\end{array}$ & $\mathrm{ND} / 35$ & ND/ Normal \\
\hline 7 & 68 & F & II & $\begin{array}{l}\text { Pantoprazole, rosuvastatin, } \\
\text { paroxetine, domperidone, } \\
\text { lebrikizumab }\end{array}$ & $\begin{array}{l}\text { Phototoxicity } \\
\text { (Grade II) on neck, } \\
\text { upper chest, dorsa of } \\
\text { forearms and hands }\end{array}$ & 7 & 2403 & $\begin{array}{l}\text { Topical } \\
\text { corticosteroids, } \\
\text { photoprotective } \\
\text { measures, } \\
\text { PFD continued }\end{array}$ & ND/Normal: 1.75 & ND & Erythema & $\begin{array}{l}\text { NI :- } \\
\text { UVA : ++ }\end{array}$ & $\mathrm{ND} / 42$ & ND/Normal \\
\hline 8 & 66 & $\mathrm{M}$ & II & None & $\begin{array}{l}\text { Phototoxicity } \\
\text { (Grade II on } \\
\text { head and dorsa of } \\
\text { hands }\end{array}$ & 2 & 2403 & $\begin{array}{l}\text { Topical } \\
\text { corticosteroids, } \\
\text { PFD discontinued }\end{array}$ & ND & ND & ND & ND & ND & ND \\
\hline
\end{tabular}

y: years. M: male. F: female. Grade II: according to the Common Terminology Criteria Adverse Event (CTCAE). PFD: pirfenidone. MED: minimal erythema dose. ND: not done. NI: non-irradiated PFD patch test. - : negative reaction. + or ++ : positive reaction according International Contact Dermatitis Research Group (ICDRG) system.

han six months

** In our laboratory, the normal values for UVA MED were 21-80 J/cm2. MED was considered to be pathological after positive reaction in response to $20 \mathrm{~J} / \mathrm{cm}^{2}, 24 \mathrm{hours}$ after UVA exposure. 